\title{
„Jede Praxis sollte mit der GOZ arbeiten können"
}

\author{
Mit der GOZ kennt sich FVDZ-Bundesvorstandsmitglied Dr. Christian Öttl so gut aus wie kaum \\ ein anderer. Mit weiteren Kennern der Materie sitzt er seit Anfang 2015 im GOZ-Expertenrat des \\ FVDZ. Sein Credo: Wir müssen die komplexe Gebührenordnung herunterbrechen, damit jeder sie \\ versteht und umsetzen kann.
}

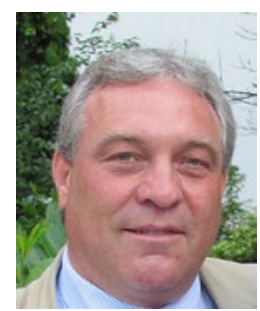

\section{Wo stand der FVDZ, als Sie den Bereich GOZ übernommen haben?}

Als ich 2011 in den Bundesvorstand des FVDZ kam, stand die „neue“ GOZ kurz vor ihrer Einführung. Der Widerstand dagegen war fehlgeschlagen. Die GOZ kam - und wir mussten mit ihr arbeiten. Was wir beim Verband versuchten, war, unsere Mitglieder möglichst fit zu machen, um mit der Gebührenordnung arbeiten zu können. Wir haben schon mit der Einführung über das gesamte Land Informationsveranstaltungen auf Basis meines Mustervortrages durchgeführt. Anschließend haben wir einen GOZ-Navigator entwickelt, der zu GOZ-Positionen Steigerungsfaktoren aufzeigte, die bei vergleichbaren BEMA-Positionen gleiche Honorare bedeuten würden. Das änderte aber nichts an der grundsätzlichen Kritik, die wir hatten: Die Punktwerte wurden quasi seit 1965 nicht erhöht und die Zahnärzte von der wirtschaftlichen Entwicklung in Deutschland abgekuppelt. Was aber wirklich dramatisch ist: Die moderne Zahnheilkunde wurde in der neuen GOZ nicht abgebildet. Es bleibt bei der analogen Berechnung fehlender Leistungen. Diese wurde für die Kollegenschaft intensiv im Praxishandbuch des FVDZ aufgearbeitet

\section{Was haben Sie in der zurückliegenden Wahlperiode erreicht?}

Der FVDZ-Bundesvorstand hat mit der neuen Legislatur seine Ziele neu justiert. Es war klar, dass eine Totalverweigerung gegen diese GOZ nicht zielführend ist. Wir müssen mit ihr arbeiten und wollen für die Kollegenschaft einen rechtssicheren Rahmen aufzeigen. Dafür haben wir den GOZ-Expertenrat aus der Taufe gehoben, der sich sehr intensiv mit der GOZ und ihren Möglichkeiten auseinandersetzt. Dieses Wissen geben wir weiter, möglichst breit gestreut, wie beispielsweise in der Artikelserie zum „Gangbaren Weg“ im DFZ oder in Vorträgen. Damit bringen wir den Zahnärzten das Handwerkzeug: Wie arbeite ich mit Ergänzungsleistungen, wie zeige ich dem Patienten die besseren Alternativen, wie gehe ich mit den Schnittstellen zwischen BEMA und GOZ um - und vor allem: Wie geht das alles rechtssicher. Der GOZExpertenrat ist noch jung, aber mit vollem Engagement dabei, das ist super angelaufen. Unabhängig davon bleiben wir bei unserer Kritik, dass eine deutliche Punktwerterhöhung stattfinden muss und eine jährliche Erhöhung des Punktwertes unumgänglich ist. Diese Forderungen sind zum Teil sogar von Herrn Seehofer im Bundesrat eingebracht worden und nicht eingeflossen. Von daher brauchen wir einen langen Atem.

\section{Was ist das Ziel für die nächste Wahlperiode?}

Wir stellen uns darauf ein, dass wir in den nächsten Jahren mit einer unveränderten GOZ arbeiten müssen. Deshalb ist es uns wichtig, die Aufklärung der Kollegenschaft zu intensivieren. Deshalb starten wir mit einem Konzept, das die Kollegen an unterschiedlichen Punkten im Berufsleben abholt, um ihnen die GOZ, ihre Möglichkeiten und Feinheiten nahezubringen. Es wird Vortragsreihen für Studenten geben, in denen sie zum ersten Mal mit Gebührenordnungen und Wirtschaftlichkeit in der Praxis konfrontiert werden. Den Zahnärzten, die kurz vor der Niederlassung stehen oder sich gerade niedergelassen haben, müssen wir andere Dinge an die Hand geben. Diese brauchen eine gute Erstaufarbeitung der GOZ. Weiter werden wir je nach Zielgruppe Optimierungspotenziale aufzeigen. Alles mit dem Ziel, dass jede Praxis mit der GOZ umgehen kann und nicht nur Experten.

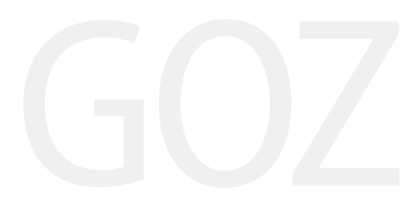

